\title{
Pump-tuning optical parametric oscillation and sum-frequency mixing with KTP pumped by a Ti:sapphire laser
}

\author{
J.Q. Yao ${ }^{\text {a }}$, X. Ding ${ }^{\text {a }}$, J.Y. Qiao ${ }^{\text {a }, \text { C.C. Yang }}{ }^{\text {b** }}$, I.J. Hsu ${ }^{\text {b }, \text { C.W. Hsu }}{ }^{\text {b }}$

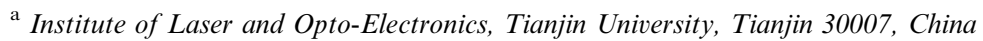 \\ ${ }^{\mathrm{b}}$ Department of Electrical Engineering, Graduate Institute of Electro-Optical Engineering and Graduate Institute of \\ Communication Engineering, National Taiwan University, 1, Roosevelt Road, Sec. 4, Taipei 106, Taiwan, ROC
}

Received 8 November 2000; received in revised form 26 February 2001; accepted 3 April 2001

\begin{abstract}
We report the implementation of a KTP optical parametric oscillator pumped by a pulsed Ti:sapphire laser. Including signal and idler, it could be continuously tuned from 1.261 through $2.532 \mu \mathrm{m}$ by varying the pump wavelength. Two major improvements were achieved, including the connection of the signal and idler tuning ranges and the high output pulse energy through the signal and idler tuning ranges. The first improvement was achieved by discovering two particular sets of phase-matching angles. The second improvement was realized by using five sets of resonator mirrors for oscillating either signal or idler. Theoretical calculations were also conducted for the effective nonlinear coefficient, walk-off angle, phase-matching acceptance angle, and output spectral widths for the concerned phase-matching angles. The second part of this paper presents the experimental and theoretical results of sum-frequency mixing (SFM) of a Ti:sapphire laser and a $1.064 \mu \mathrm{m} \mathrm{Nd:YAG} \mathrm{laser.} \mathrm{By} \mathrm{using} \mathrm{two} \mathrm{KTP} \mathrm{crystals} \mathrm{cut} \mathrm{at} \theta=76^{\circ}$ and $85^{\circ}\left(\varphi=90^{\circ}\right.$ in both crystals), respectively, we have experimentally achieved SFM tuning range from 459.3 through $472.9 \mathrm{~nm}$. The energy conversion efficiencies were reasonably high. (c) 2001 Published by Elsevier Science B.V.
\end{abstract}

Keywords: Optical parametric oscillator; Sum-frequency mixing; Ti:sapphire laser; KTP crystal

\section{Introduction}

A Ti:sapphire laser is the most widely tunable laser in the current laser technology. It has a tuning range from 0.68 through $1.1 \mu \mathrm{m}$. Nonlinear wavelength conversion pumped with a Ti:sapphire laser has been reported in literature. With the pumping of a nanosecond Ti:sapphire laser, Rines

\footnotetext{
${ }^{*}$ Corresponding author. Tel.: +886-2-2365-7624; fax: $+886-$ 2-2365-2637.

E-mail address: ccy@cc.ee.ntu.edu.tw (C.C. Yang).
}

et al. have implemented second-harmonic generation (SHG) at $462 \mathrm{~nm}$ with type-I phase matching in $\mathrm{BBO}$, third-harmonic generation at $308 \mathrm{~nm}$ with types-I and II phase matching in BBO, fourthharmonic generation at $220 \mathrm{~nm}$ in $\mathrm{BBO}$ and $\mathrm{LBO}$, sum-frequency mixing (SFM) at $194 \mathrm{~nm}$ in LBO, and optical parametric oscillation for up to $3 \mu \mathrm{m}$ with type-II noncritical phase matching in KTP, KTA, and CTA crystals [1]. Also, Bartoshevich et al. reported their results of large tuning range from 0.43 through $2.3 \mu \mathrm{m}$ with $\mathrm{SHG}$ and difference frequency mixing (DFM), pumped with a dualwavelength Ti:sapphire laser [2]. This large tuning 
range consists of three blocks: the fundamental wavelength range (Ti:sapphire laser, $0.68-$ $1.05 \mu \mathrm{m})$, the SHG $(0.34-0.525 \mu \mathrm{m})$, and the two sections of DFM $(0.503-0.79$ and $0.83-2.3 \mu \mathrm{m})$. Meanwhile, Zenzie and Perkins presented the results of single-wavelength SFM of a Ti:sapphire laser and a Nd:YAG laser to obtain a $455 \mathrm{~nm}$ light source [3]. Noncritically phase-matched KTP optical parametric oscillators (OPOs) pumped by tunable Ti:sapphire lasers were also reported. By tuning the pump wavelength, a cw signal range of $1.05-1.37 \mu \mathrm{m}$ and an idler range $2.2-3.1 \mu \mathrm{m}$ were obtained [4]. Another implementation of noncritically phase-matched KTP OPO provided a pulsed signal range of 1.03-1.28 $\mu \mathrm{m}$ and an idler range of 2.18-3.03 $\mu \mathrm{m}$ [5]. Although their tuning ranges are quite large and there is no walk-off problem with noncritical phase matching, the signal and idler wavelength ranges are disconnected. In other words, continuous tuning from near infrared through mid-infrared is not allowed with KTP pumped with Ti:sapphire laser. Also, their singly resonant oscillators for signal resulted in quite weak output power for idler. Although continuous OPO tuning for near-IR light source was implemented with $\mathrm{AgGaSe}_{2}$ crystal [6], which was cut for around $700 \mathrm{~nm}$ pump wavelength, similar implementation was never reported on KTP pumped with Ti:sapphire laser.

In this paper, we report our results of a continuously tunable KTP OPO pumped by a pulsed Ti:sapphire laser. The Ti:sapphire laser was pumped with the second harmonic of a Q-switched $\mathrm{Nd}$ :YAG laser. By choosing appropriate phasematching angles, we could connect the signal and idler wavelength ranges for continuous tuning from 1.261 through $2.532 \mu \mathrm{m}$. Also, because we used five sets of resonator mirrors of different coatings for oscillating different bands of signal and idler, the OPO output pulse energy could exceed $8.4 \mathrm{~mJ}$ in the whole tuning range. Meanwhile, our results of pump-tuning SFM of the Ti:sapphire laser and its pumping Nd:YAG laser with reasonably high energy conversion efficiencies are also reported. By using two KTP crystals of appropriate cut angles, a tuning range of 459.3-472.9 $\mathrm{nm}$ was obtained. In Section 2 of this paper, we describe the theoretical calculations of optimum phase-matching angles and related parameters of KTP for continuous OPO tuning. Then, in Section 3 , the experimental procedures and results of the pump-tuning OPO are presented. In Section 4, theoretical calculations for SFM in KTP are described. The procedures and results of SFM are discussed in Section 5. Finally, conclusions are drawn in Section 6. Note that in this paper, the signal (idler) is referred to the short (long) wavelength component in optical parametric process. The oscillating wavelength of an OPO can be either signal or idler.

\section{Theoretical calculations for OPO phase-match- ing angles}

Methods for calculating the phase-matching parameters, including phase-matching curve, effective nonlinear coefficient, acceptance angle, walk-off angle, and acceptance wavelength, of a biaxial nonlinear crystal, such as KTP, have been reported [7,8]. Detailed information can be obtained from SNLO nonlinear optics code [9]. In the calculations described below, we first searched for type-II phase-matching angles $(\theta$ and $\varphi)$ of KTP for connecting the signal and idler wavelength ranges in optical parametric process. With these angles, continuous tuning across the signal and idler ranges becomes possible. Fig. 1(a) and (b) show the signal and idler wavelength variations as the pump wavelength tuned from 0.7 through 1 $\mu \mathrm{m}$ for two sets of phase-matching angles. Parts (a) and (b) correspond to the sets of $\theta=62.5^{\circ}$, $\varphi=0^{\circ}$, and $\theta=53^{\circ}, \varphi=90^{\circ}$, respectively. In the case of part (a), the OPO tuning range, including signal and idler, covers from 1.255 through $2.546 \mu \mathrm{m}$. As shown in Fig. 2, to achieve such a continuously, broadly tunable range with angle tuning of KTP, pumped by a laser of a fixed wavelength at $0.8 \mu \mathrm{m}$, the $\theta$ angle needs to be rotated from $53^{\circ}$ through $73^{\circ}$ (assuming $\varphi=0^{\circ}$ ). For this purpose, more than one KTP crystal is usually required. From part (a) of Fig. 1, one can read that at $0.7(0.98) \mu \mathrm{m}$ pump wavelength, the signal wavelength is 1.255 (1.593) $\mu \mathrm{m}$ and the idler wavelength is $1.582(2.546) \mu \mathrm{m}$. Therefore, by tuning the pump wavelength from 0.7 through 

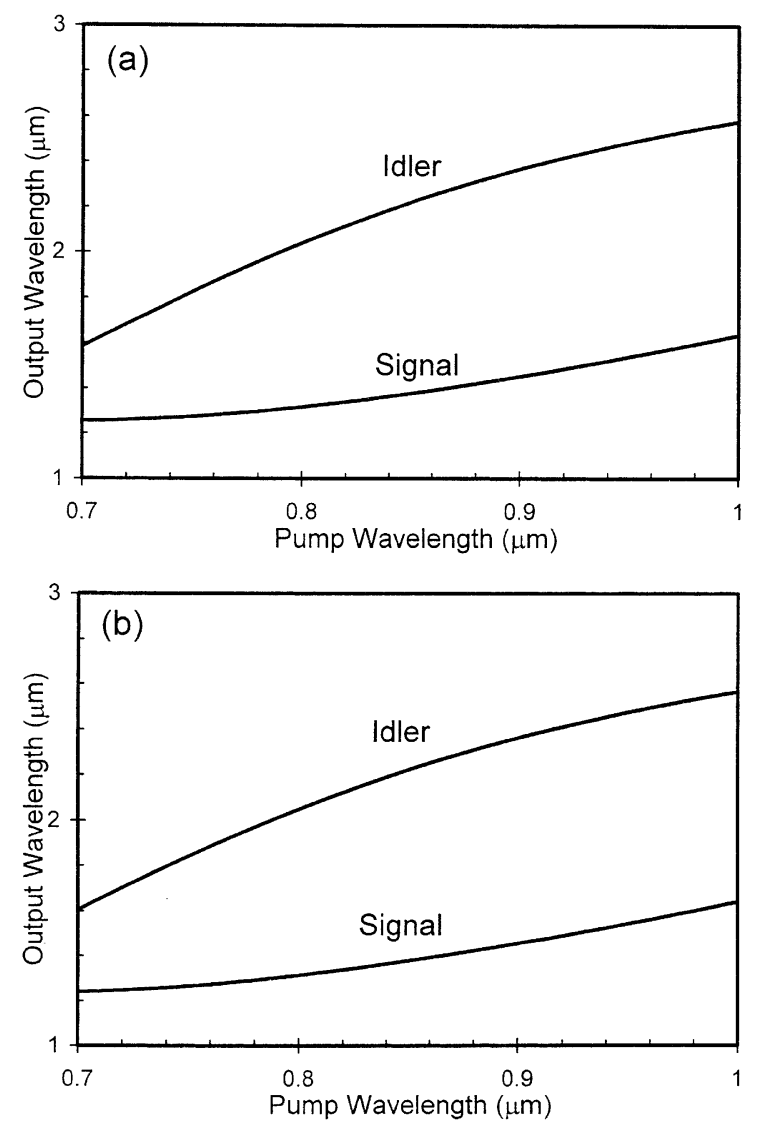

Fig. 1. Tuning curves of the signal and idler by varying pump wavelength when (a) $\theta=62.5^{\circ}, \varphi=0^{\circ}$, and (b) $\theta=53^{\circ}$, $\varphi=90^{\circ}$, respectively.

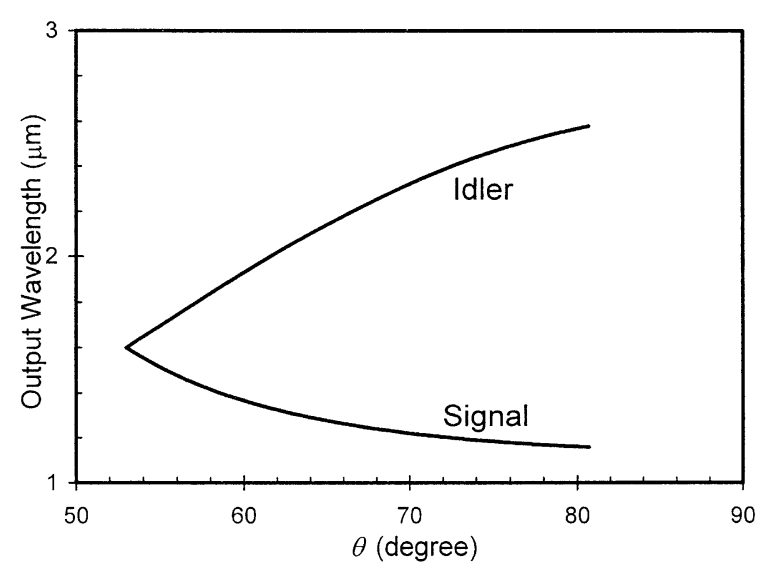

Fig. 2. Tuning curves of the signal and idler by varying KTP phase-matching angle $\theta$ when the pump wavelength is fixed at $0.8 \mu \mathrm{m}$ and $\varphi=0$.
$0.98 \mu \mathrm{m}$, we can obtain a continuous output spectrum from 1.255 through $2.546 \mu \mathrm{m}$. Similar results can be seen in the case of Fig. 1(b).

Next, we calculate the effective nonlinear coefficient, $d_{\text {eff }}$, for the two sets of phase-matching angles. Fig. 3(a) and (b) shows the results, corresponding to the cases of Fig. 1(a) and (b), respectively. Comparison between the two parts of Fig. 3, one can find that the effective nonlinear coefficient is larger with the angles: $\theta=62.5^{\circ}$, $\varphi=0^{\circ}$. In this case, $d_{\text {eff }}$ varies from 6.592 through $6.598 \mathrm{pm} / \mathrm{V}$ as the pump wavelength is tuned from 0.7 through $0.98 \mu \mathrm{m}$. These values are slightly smaller than that in the case of noncritical phase
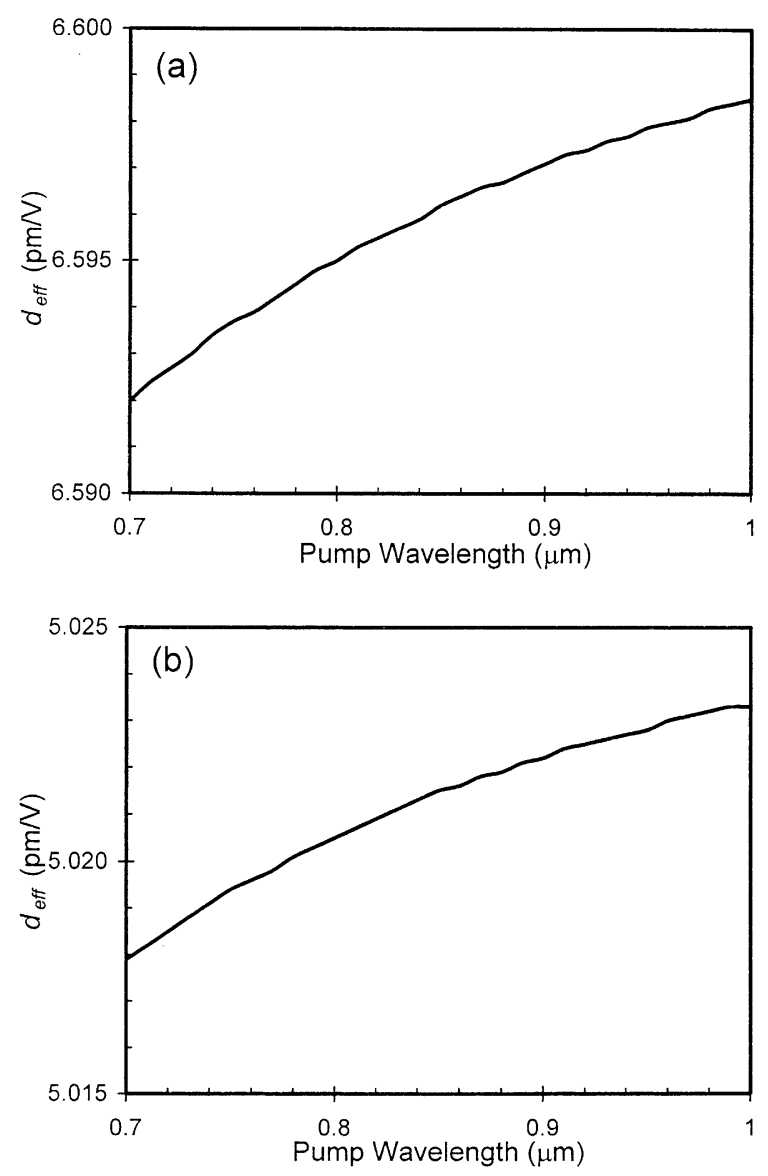

Fig. 3. Effective nonlinear coefficient, $d_{\mathrm{eff}}$, as a function of pump wavelength when (a) $\theta=62.5^{\circ}, \varphi=0^{\circ}$, and (b) $\theta=53^{\circ}$, $\varphi=90^{\circ}$, respectively. 


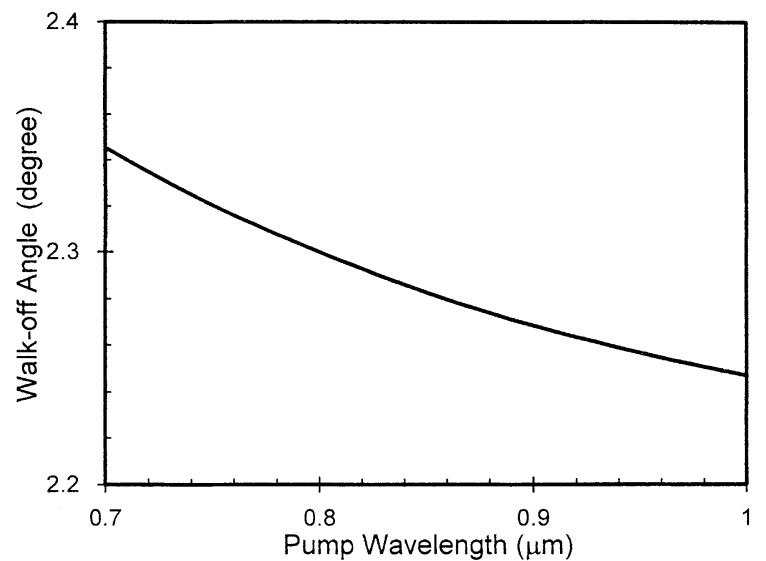

Fig. 4. Walk-off angle dependence on pump wavelength when $\theta=62.5^{\circ}, \varphi=0^{\circ}$.

matching $\left(\theta=90^{\circ}, \varphi=90^{\circ}\right)$, which is $7.6 \mathrm{pm} / \mathrm{V}$. The variation of walk-off angle between the signal and idler in the same case is shown in Fig. 4. This angle varies between $2.25^{\circ}$ and $2.35^{\circ}$. Note that in this case, there is no walk-off between the pump and signal.

The acceptance angle for efficient nonlinear conversion is usually defined as the angular ranges $\Delta \theta$ and $\Delta \varphi$ such that the wave number mismatch $\Delta k$ satisfies $|\Delta k| \leqslant \pi / l$. Here, $l$ is the crystal length. With the aforementioned two sets of phasematching conditions, $\Delta \theta l$ and $\Delta \varphi l$ are plotted as functions of pump wavelength in Figs. $5(\theta=$ $\left.62.5^{\circ}, \varphi=0^{\circ}\right)$ and $6\left(\theta=53^{\circ}, \varphi=90^{\circ}\right)$. Both parts (a) of these two figures show that $\Delta \theta l$ is always between 10 and $18 \mathrm{rad} \mu \mathrm{m}$. Also, both parts (b) of the two figures show that $\Delta \varphi l$ is always between 240 and $340 \mathrm{rad} \mu \mathrm{m}$. The acceptance angles correspond to the acceptance conversion wavelength ranges or the spectral widths $(\Delta \lambda)$ of the signal and idler. The variations of $\Delta \lambda l$ of the signal and idler are shown in Fig. 7(a) $\left(\theta=62.5^{\circ}, \varphi=0^{\circ}\right)$ and (b) $\left(\theta=53^{\circ}, \varphi=90^{\circ}\right)$. These results were obtained by assuming that the pump spectral width was zero. If there is a finite pump spectral width, the spectral widths of the signal and idler can be obtained by the superposition of the contributions from different components of pump frequency. Because mode competition exists inside an oscillator, the results of the superposition can only be
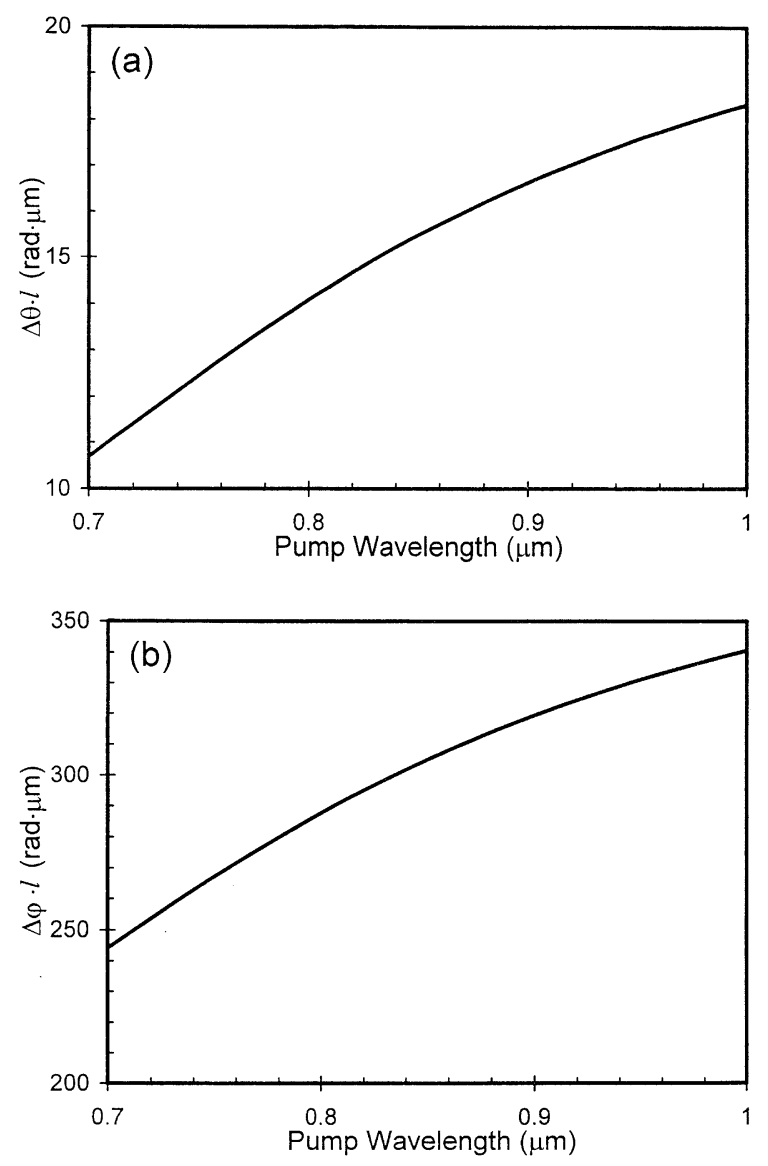

Fig. 5. Phase-matching acceptance angles multiplied by the crystal length, $\Delta \theta l$ (a) and $\Delta \varphi l$ (b), as functions of pump wavelength when $\theta=62.5^{\circ}, \varphi=0^{\circ}$.

regarded as the upper bounds of the output spectral widths of the signal and idler. Parts (a) $(\theta=$ $\left.62.5^{\circ}, \varphi=0^{\circ}\right)$ and (b) $\left(\theta=53^{\circ}, \varphi=90^{\circ}\right)$ of Fig. 8 shows these upper bounds of line width for the two phase-matching conditions when $l$ is $10 \mathrm{~mm}$ and the pump spectral width is $1 \mathrm{~nm}$. With pump wavelength fixed at $0.8 \mu \mathrm{m}$ and $l$ at $10 \mathrm{~mm}$, the maximum line widths are plotted as functions of pump line width in Fig. 9. The comparisons between Figs. 7-9 reveal that when the pump line width is smaller than $0.1 \mathrm{~nm}$, the signal and idler line widths are mainly controlled by their acceptance angles; while the pump line width is larger than $0.1 \mathrm{~nm}$, they are essentially determined by the pump line width. 

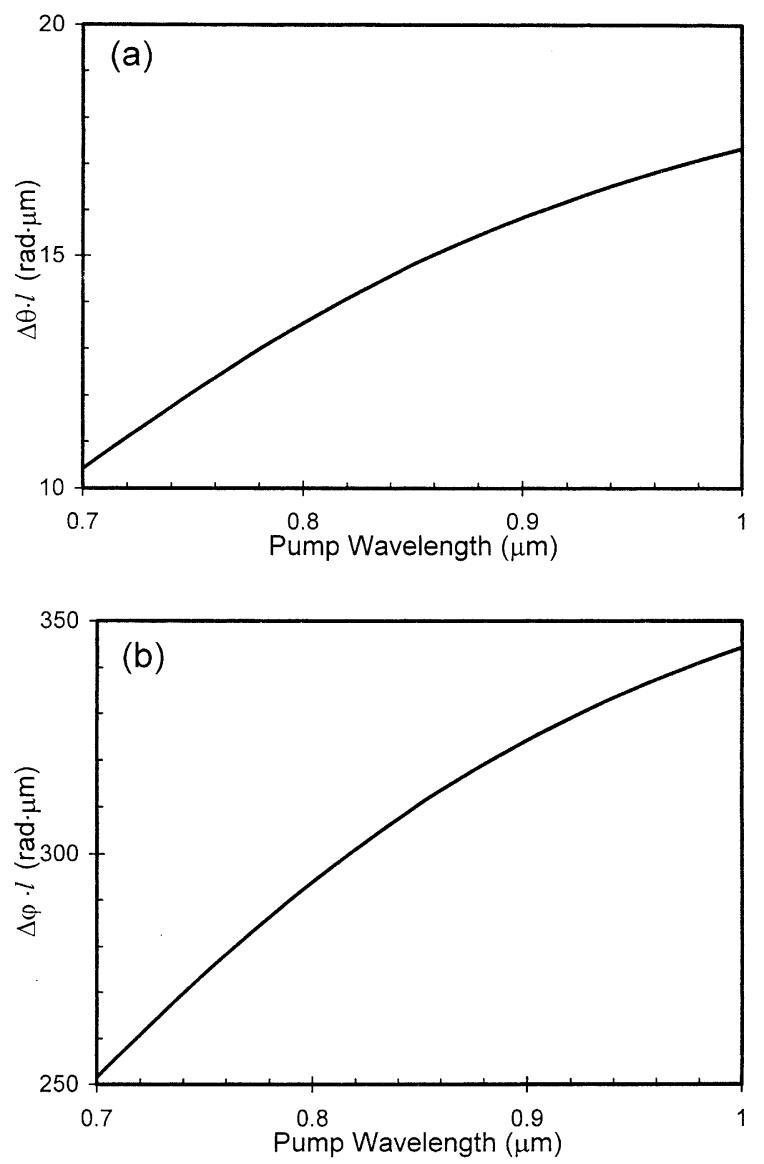

Fig. 6. Phase-matching acceptance angles multiplied by the crystal length, $\Delta \theta l$ (a) and $\Delta \varphi l$ (b), as functions of pump wavelength when $\theta=53^{\circ}, \varphi=90^{\circ}$.

\section{Experimental procedures and results of OPO}

The OPO setup is sketched in Fig. 10, in which the pulsed Ti:sapphire laser, tunable from 0.7 through $0.98 \mu \mathrm{m}$, is pumped by a Q-switched (10 $\mathrm{Hz}$ ), frequency-doubled Nd:YAG laser. The Ti: sapphire laser could provide pulses with pulse energy from $76.2 \mathrm{~mJ}$ (at $795 \mathrm{~nm}$ ) to $31 \mathrm{~mJ}$ (at 980 $\mathrm{nm}$ ), line width around $1.1 \mathrm{~nm}$, and output divergence angle about $1.2 \mathrm{mrad}$. With type-II phase matching, the KTP crystal was cut for $\theta=62.5^{\circ}$, $\varphi=0^{\circ}$, which corresponded to one of the phasematching conditions described in Section 2. The KTP crystal had the dimensions of $5 \mathrm{~mm} \times$ $5.7 \mathrm{~mm} \times 8.6 \mathrm{~mm}$. There was no coating on either
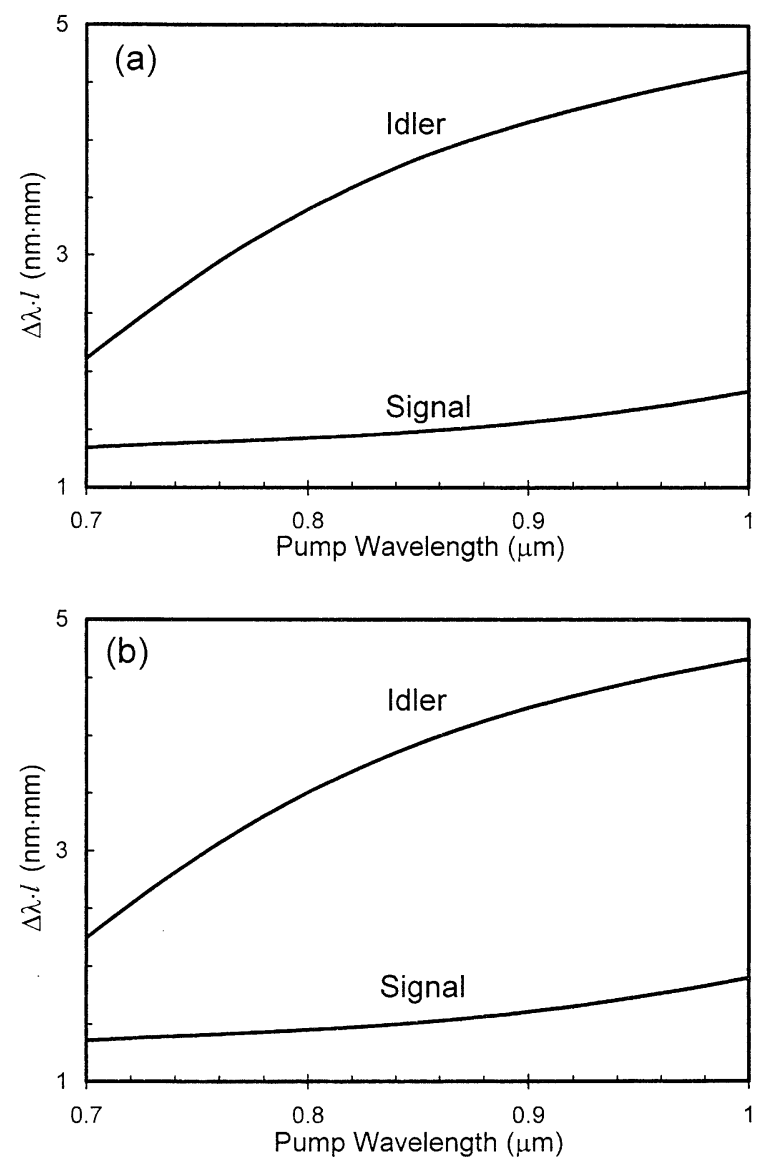

Fig. 7. Phase-matching acceptance spectral widths multiplied by the crystal length, $\Delta \lambda l$ when (a) $\theta=62.5^{\circ}, \varphi=0^{\circ}$, and (b) $\theta=53^{\circ}, \varphi=90^{\circ}$, respectively.

end face of the crystal. The OPO resonator had a flat-flat configuration. To obtain sufficiently high output pulse energy for both signal and idler, five sets of mirrors with high reflectivity wavelength ranges of $1.2-1.5,1.49-1.8,1.79-2.1,2.09-2.4$, and $2.39-2.6 \mu \mathrm{m}$ were used for the resonator. The signal reflectivity levels of the end mirrors (highly transmitted for Ti:sapphire laser, denoted with " 3 ", in Fig. 10) of the resonator were always higher than $99 \%$. Those of the output couplers (also highly transmitted for Ti:sapphire laser, denoted with " 5 " in Fig. 10) were between $89 \%$ and $92 \%$. The cavity length was $34 \mathrm{~mm}$. The Ti:sapphire pump beam was focused with a lens of $500 \mathrm{~mm}$ in focal length into a spot size of $2.3 \mathrm{~mm}$ in diameter. 

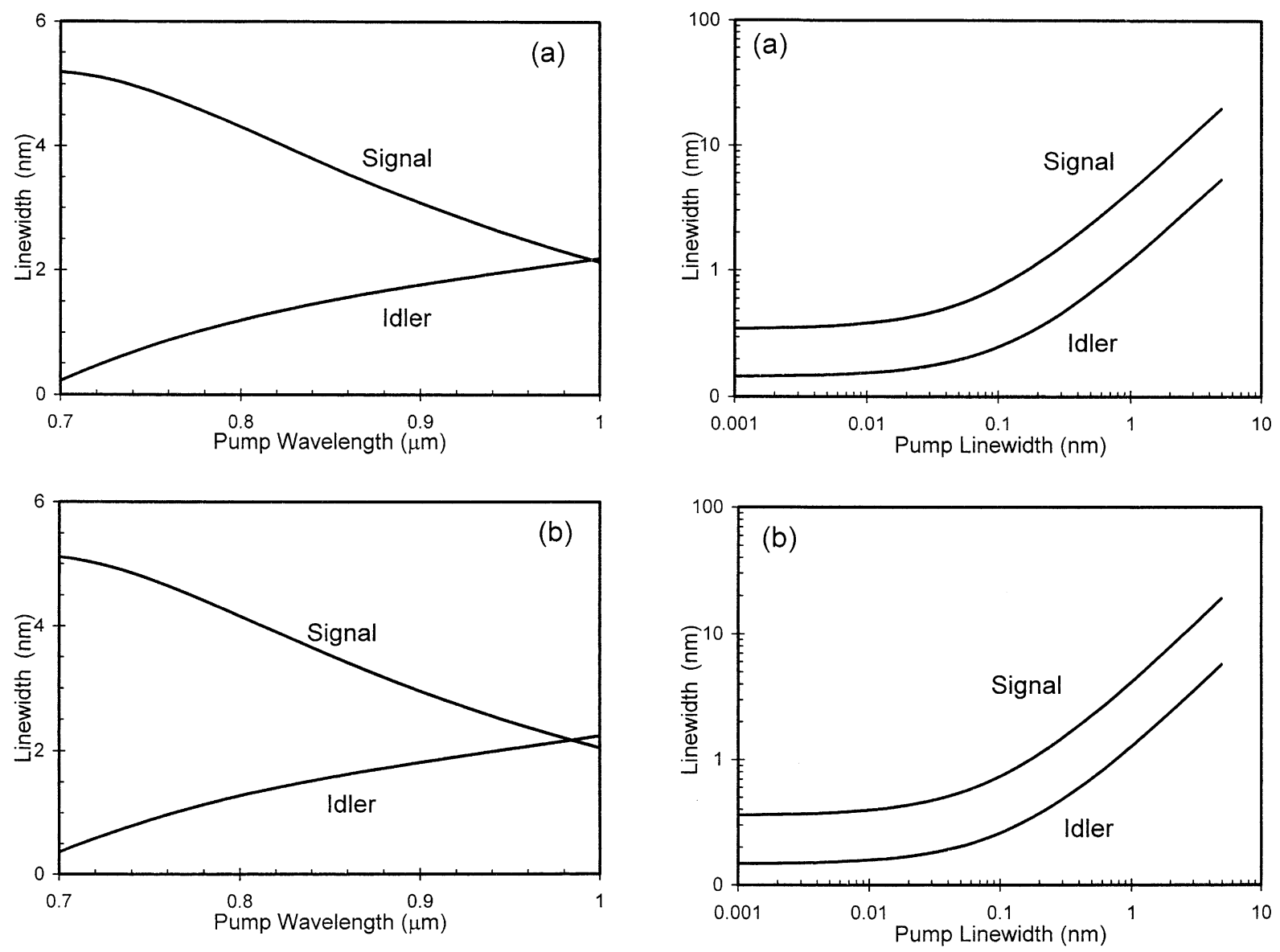

Fig. 8. Possibly maximum line widths as functions of pump wavelength when the KTP crystal is $10 \mathrm{~mm}$ in length, pump line width is $1 \mathrm{~nm}$, and (a) $\theta=62.5^{\circ}, \varphi=0^{\circ}$, and (b) $\theta=53^{\circ}$, $\varphi=90^{\circ}$, respectively.

The focal point was located $1.4 \mathrm{~mm}$ behind the KTP crystal to avoid crystal damage. The linear polarization of the pump was along the $y$-axis of the KTP crystal. The two short-wavelength mirror sets were used to oscillate the signal and the three long-wavelength mirror sets were used for oscillation of the idler. Such combinations guarantee sufficiently high output pulse energy for both signal and idler. Therefore, the experimental results discussed below always refer to those of the oscillating wavelength, either signal or idler.

Combining the results of the five mirror sets, we obtained the tuning curves shown in Fig. 11. Here, the empty circles represent experimental data and

Fig. 9. Possibly maximum line widths as functions of pump line width when the KTP crystal is $10 \mathrm{~mm}$ in length, pump wavelength is $0.8 \mu \mathrm{m}$, and (a) $\theta=62.5^{\circ}, \varphi=0^{\circ}$, and (b) $\theta=53^{\circ}$, $\varphi=90^{\circ}$, respectively.

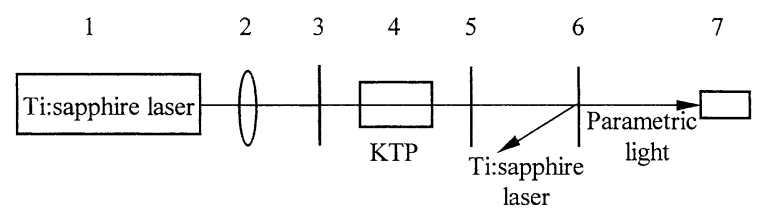

Fig. 10. Experimental setup for the pump-tuning KTP OPO. Components include: 1, Ti:sapphire laser (700-980 nm); 2, lens (focal length $=500 \mathrm{~mm}$ ); 3 , end mirror of the OPO; 4, KTP crystal; 5 , output coupler of the OPO; 6 , dichroic mirror; 7 , detector.

the continuous curves stand for theoretical predictions. With the pump laser tuned from 0.7 through $0.98 \mu \mathrm{m}$, signal wavelengths from 1.261 


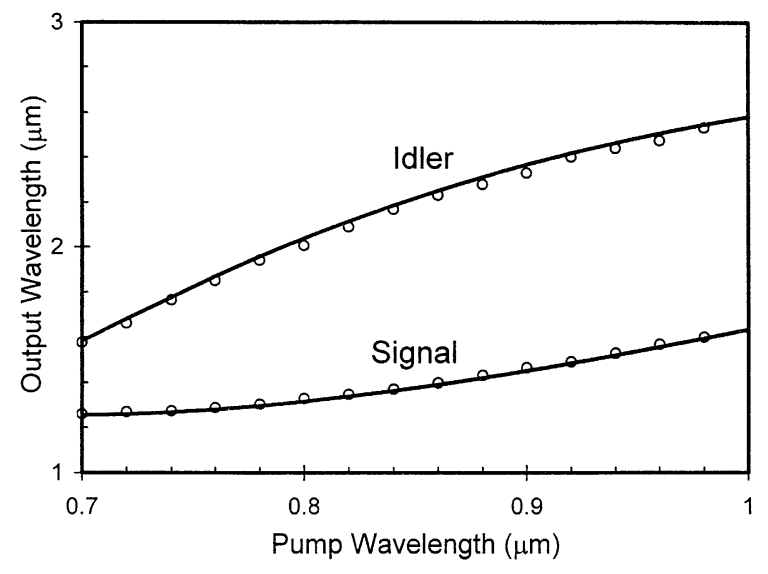

Fig. 11. Experimental $(\bigcirc)$ and theoretical $(-)$ results of the tuning curves of the OPO with the KTP crystal cut at $\theta=62.5^{\circ}$, $\varphi=0^{\circ}$.

through $1.599 \mu \mathrm{m}$ (with the two short-wavelength mirror sets) and idler wavelengths from 1.576 through $2.532 \mu \mathrm{m}$ (with the three long-wavelength mirror sets) were achieved. The typical oscillating output line width was around $1.7 \mathrm{~nm}$ The experimental and theoretical results in Fig. 11 agree well except a 10-14 $\mathrm{nm}$ discrepancy. The discrepancy may originate from the slight inaccuracies of the Sellmeier equation for KTP and the cutting angles of the crystal. In the whole tuning range $(1.261-2.532 \mu \mathrm{m})$, the oscillating output pulse energy could always exceed $8.4 \mathrm{~mJ}$ with the pump pulse energy of $41.3 \mathrm{~mJ}$ at $795 \mathrm{~nm}$ (pump pulse energy dropped as the wavelength moved away from this value). In the tuning range, the OPO conversion efficiency was between $28 \%$ and $35.7 \%$.

\section{Calculations of phase-matching parameters for SFM}

Previous studies have shown that to satisfy the phase-matching conditions for SFM of a Ti:sapphire laser and a Nd:YAG laser at $1.064 \mu \mathrm{m}$, the wavelength of the Ti:sapphire laser must be longer than $0.808 \mu \mathrm{m}$ [10]. Figs. 12 and 13 show the SFM tuning curves in two KTP planes of $\theta=90^{\circ}$ and $\varphi=90^{\circ}$, respectively, with type-II(A) phase

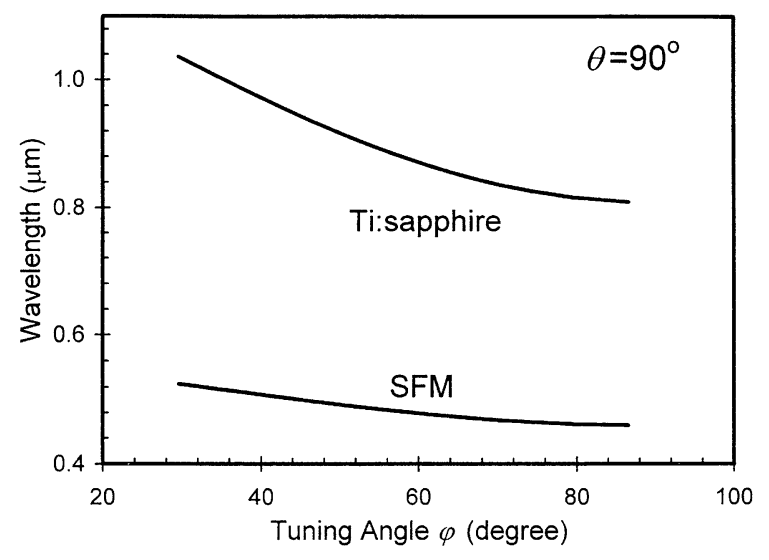

Fig. 12. Tuning curve of the SFM (type-II(A) phase matching) of a Ti:sapphire laser and a $1.064 \mu \mathrm{m} \mathrm{Nd:YAG} \mathrm{laser} \mathrm{when} \theta$ is fixed at $90^{\circ}$. The tuning range of the Ti:sapphire laser is also plotted.

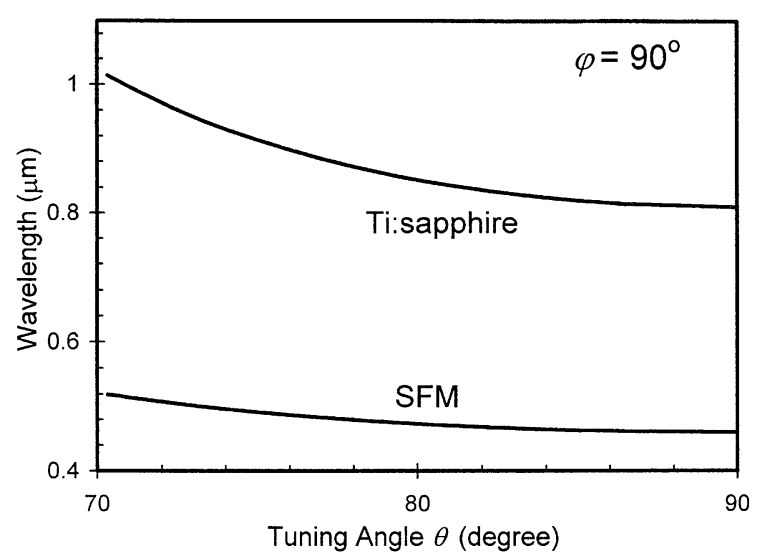

Fig. 13. Tuning curve of the SFM (type-II(A) phase matching) of a Ti:sapphire laser and a $1.064 \mu \mathrm{m} \mathrm{Nd:YAG} \mathrm{laser} \mathrm{when} \varphi$ is fixed at $90^{\circ}$. The tuning range of the Ti:sapphire laser is also plotted.

matching. In the two figures, the upper curves depict the mixed Ti:sapphire wavelength and the lower curves depict the output wavelengths of SFM, as functions of the other phase-matching angle. Fig. 12 shows that with $\theta$ fixed at $90^{\circ}, \varphi$ must be changed from $38^{\circ}$ to $87^{\circ}$ (totally $49^{\circ}$ ) for phase-matching Ti:sapphire laser wavelength from 0.809 through $0.98 \mu \mathrm{m}$ to give SFM wavelength from 0.459 through $0.51 \mu \mathrm{m}$. On the other hand, Fig. 13 shows that with $\varphi$ fixed at $90^{\circ}$, for tuning 


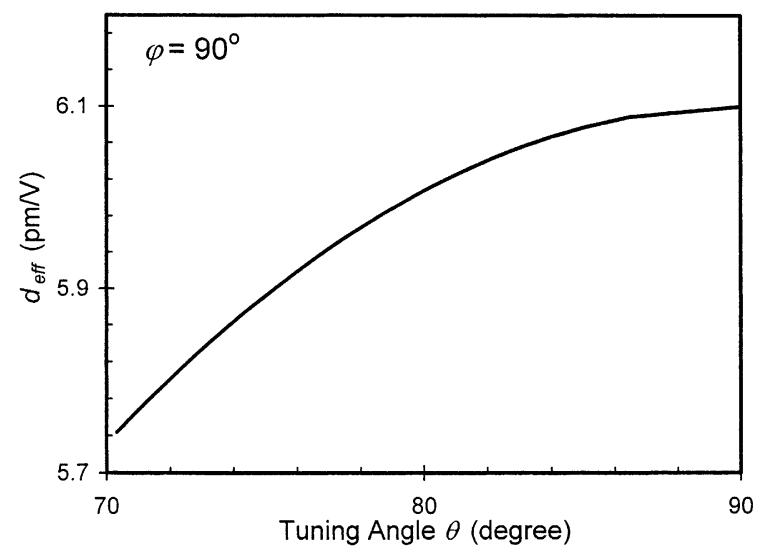

Fig. 14. Effective nonlinear coefficient, $d_{\text {eff }}$, of the SFM as a function of tuning angle $\theta$ when $\varphi$ is fixed at $90^{\circ}$.

the same range of SFM wavelength, we need only change $\theta$ from $71^{\circ}$ through $90^{\circ}$ (totally $17^{\circ}$ ). Therefore, the case of Fig. 13 is preferred in experiment. Figs. 14 and 15 show the variations of the effective nonlinear coefficient, $d_{\mathrm{eff}}$, and walk-off angle (between the sum-frequency wave and the Ti:sapphire laser) of this case. One can see that $d_{\text {eff }}$ is in the range between 5.7 and $6.1 \mathrm{pm} / \mathrm{V}$ and the walk-off angle is always smaller than $2^{\circ}$ in the concerned $\theta$ range. In the case of noncritically phase matching, i.e., $\theta=90^{\circ}$ and $\varphi=90^{\circ}, d_{\text {eff }}$

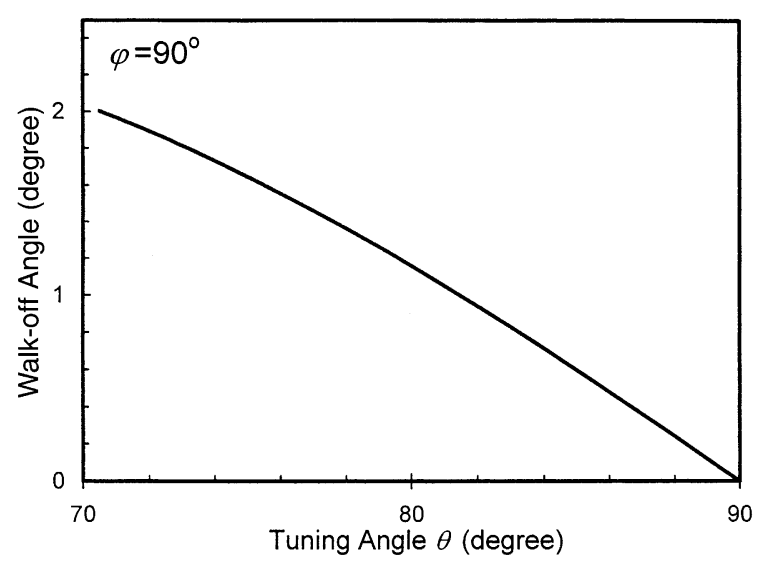

Fig. 15. Walk-off angle between the SFM wave and the Ti:sapphire laser as a function of tuning angle $\theta$ when $\varphi$ is fixed at $90^{\circ}$. reaches the maximum value and the walk-off angle is zero. However, it cannot provide the large tuning range as described in Figs. 13-15. Note that in the phase-matching conditions of Figs. 13-15, there is no walk-off between sum-frequency wave and the Nd:YAG laser.

\section{Experimental procedures and results of SFM}

We conducted the SFM of the Ti:sapphire laser and the Nd:YAG laser, whose second harmonic was used to pump the Ti:sapphire laser. The experimental setup is shown in Fig. 16, in which numbers are used to assign different optical components. Among them, numbers 1, 2, 14, and 16 refer to Nd:YAG rod, KTP crystal for SHG, Ti:sapphire crystal, and KTP crystal for SFM, respectively. To equalize the optical paths of the $1.064 \mu \mathrm{m}$ beam and the Ti:sapphire beam, a delay line, denoted by numbers $6-8$, was employed. Meanwhile, to let the two beams have about the same beam size in the SFM KTP crystal, a focusing lens (denoted by number 10) of $500 \mathrm{~mm}$ in focal length was used for the $1.064 \mu \mathrm{m}$ beam. To minimize the tuning angle, we adopted the crystal cut in the case of Figs. 13-15, i.e., $\varphi$ was fixed at $90^{\circ}$. For implementing the large tuning range as

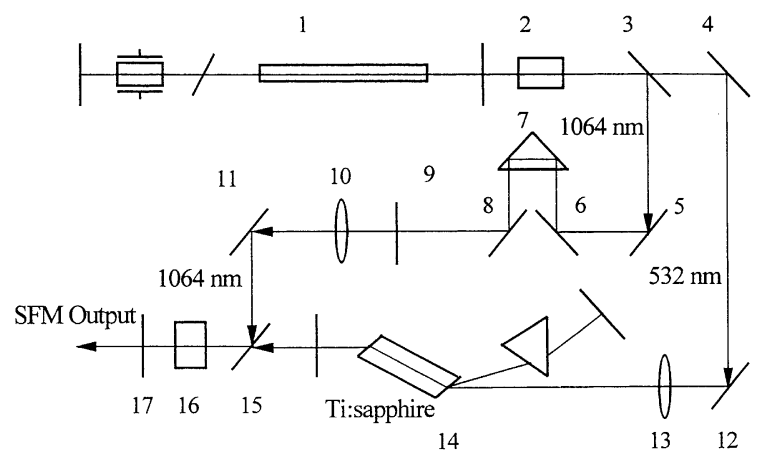

Fig. 16. Experimental setup for the SFM of a Ti:sapphire laser and a $1.064 \mu \mathrm{m} \mathrm{Nd:YAG} \mathrm{laser.} \mathrm{Optical} \mathrm{components} \mathrm{include:} \mathrm{1,}$ Nd:YAG rod; 2, KTP crystal for SHG; 3 and 15, dichroic mirrors; 4 and 12, $532 \mathrm{~nm}$ mirrors; 5, 6, 8, 11, and 15, $1064 \mathrm{~nm}$ mirrors; 7, prism; 9, $1064 \mathrm{~nm}$ attenuator; 10 and 13, focusing lenses; 14, Ti:sapphire crystal; 16, KTP crystal for SFM; 17, high-reflection mirror for $800-1100 \mathrm{~nm}$. 


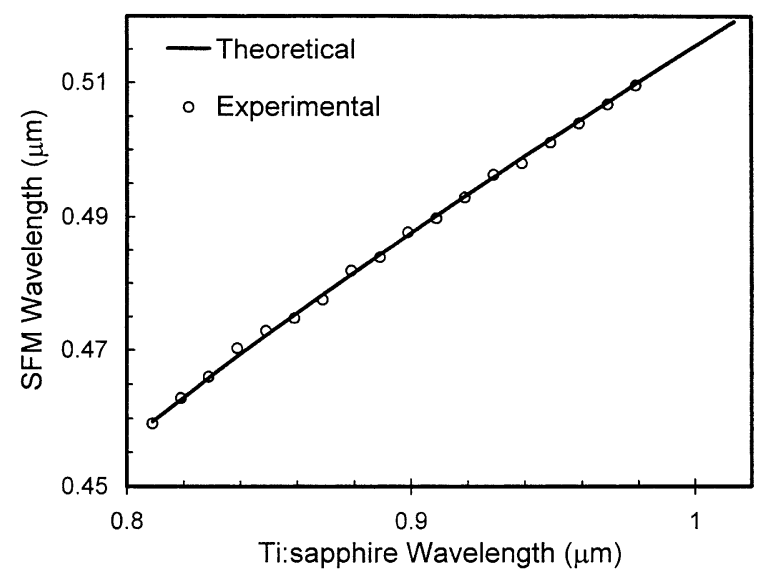

Fig. 17. Tuning curve of the SFM tuned with Ti:sapphire laser wavelength. Empty circles and continuous curve represent the experimental and theoretical results, respectively.

shown in Fig. 13, we still needed to use two KTP crystals. The two crystals were cut at $\theta=76^{\circ}$ and $85^{\circ}$, respectively. They have the same dimensions as $4 \mathrm{~mm} \times 4 \mathrm{~mm} \times 7 \mathrm{~mm}$. The crystal with $\theta=$ $85^{\circ}$ was used for mixing Ti:sapphire wavelength from 809 through $849 \mathrm{~nm}$ to produce SFM wavelength from 459.3 through $472.9 \mathrm{~nm}$ (crystal rotation from $\theta=80^{\circ}$ to $90^{\circ}$ ). The crystal with $\theta=76^{\circ}$ was used for mixing Ti:sapphire wavelength from 844 through $979 \mathrm{~nm}$ to produce SFM wavelength from 470.7 through $509.6 \mathrm{~nm}$ (crystal rotation from $\theta=72^{\circ}$ to $81^{\circ}$ ). The polarization of all three interacting waves is along the $x$-axis.

Fig. 17 shows the measured (empty circles) and predicted (continuous curve) SFM wavelength versus Ti:sapphire wavelength. The experimental data agree well with the theoretical predictions. Fig. 18 shows the results of SFM efficiency, $\eta$, over the SFM tuning range by fixing the Ti:sapphire pulse energy at $17 \mathrm{~mJ}$ and $1.064 \mu \mathrm{m}$ pulse energy at $42 \mathrm{~mJ}$. One can see two humps corresponding to the two KTP crystals. The highest efficiency was achieved around $0.464 \mu \mathrm{m}$ to give $12.34 \%$. The lowest efficiency was obtained at $0.51 \mu \mathrm{m}$ and still had a value of $7.5 \%$. Fig. 19 shows another set of conversion efficiency data, in which the Ti:sapphire laser pulse energy was varied from 10 to 48 $\mathrm{mJ}$ and its wavelength was fixed so that the SFM wavelength was fixed at $0.463 \mu \mathrm{m}$. The pulse en-

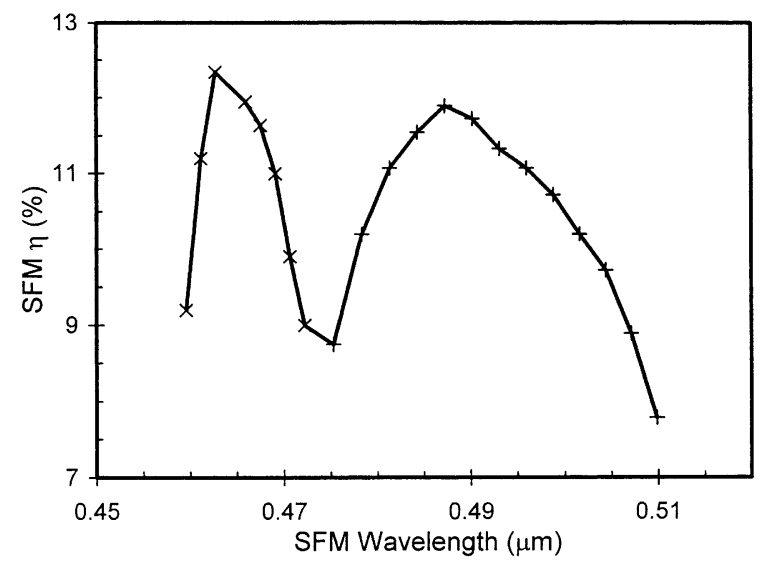

Fig. 18. SFM conversion efficiency as a function of wavelength. The data points of " $X$ " ("+") were obtained with the KTP crystal cut at $\theta=85^{\circ}\left(76^{\circ}\right)$. The curve is used to connect the data points.

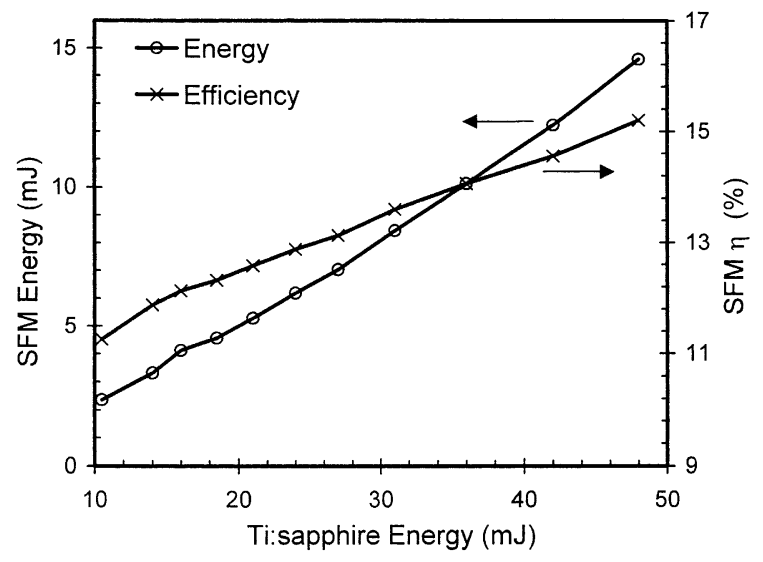

Fig. 19. Output pulse energy and energy conversion efficiency at $463 \mathrm{~nm}$ of SFM wavelength as functions of Ti:sapphire pulse energy.

ergy of $1.064 \mu \mathrm{m}$ laser was still fixed at $42 \mathrm{~mJ}$. As shown by the crosses, the efficiency varies from $11 \%$ to around $15 \%$. The maximum value is $15.2 \%$. With the empty circles, Fig. 19 also shows the variation of SFM output energy, which varies from 2 to almost $15 \mathrm{~mJ}$ with the maximum value at $14.6 \mathrm{~mJ}$. The conversion efficiency described above may not be as high as previously reported [1]. It can be increased if the KTP crystals are AR-coated and the coatings of other optical components are improved. 


\section{Conclusions}

In summary, we have implemented a KTP OPO pumped by a pulsed Ti:sapphire laser. Including signal and idler, it could be continuously tuned from 1.261 through $2.532 \mu \mathrm{m}$ by varying the pump wavelength. There are two major improvements in our OPO system. First, by considering a particular set of critical phase-matching angles $\left(\theta=62.5^{\circ}\right.$, $\varphi=0^{\circ}$ ) for KTP, signal and idler tuning ranges could be connected so that the continuous tuning range $1.261-2.532 \mu \mathrm{m}$ could be achieved by changing the Ti:sapphire laser from 0.7 through $0.98 \mu \mathrm{m}$. Second, to obtain significantly high output pulse energy through the whole tuning range, five sets of oscillator mirrors of different coatings were used for oscillate signal and idler, section by section. In the whole tuning range, the minimum output pulse energy of $8.4 \mathrm{~mJ}$ was achieved. Besides the experimental implementation, theoretical calculations were also conducted for the effective nonlinear coefficient, walk-off angle, phase-matching acceptance angle, and output spectral widths for two sets of phase-matching angles, with which pump-tuning KTP OPO (pumped by a Ti:sapphire laser) could result in continuous tuning from the signal to idler range.

The second part of this paper presents the experimental and theoretical results of SFM of a Ti:sapphire laser and a $1.064 \mu \mathrm{m} \mathrm{Nd:YAG,} \mathrm{which}$ was used to provide the source for SHG for pumping the Ti:sapphire laser. Our calculations showed that by fixing the phase-matching angle $\varphi$ at $90^{\circ}$, the other angle $\theta$ needed to be varied by $17^{\circ}$ to fully use the allowable Ti:sapphire laser tuning range for SFM with $1.064 \mu \mathrm{m}$ laser. By using two KTP crystals cut at $\theta=76^{\circ}$ and $85^{\circ}$, respectively, we have experimentally achieved SFM tuning range from 459.3 through $472.9 \mathrm{~nm}$. The energy conversion efficiencies were reasonably high, However, they can be further improved if the KTP crystals are AR-coated.

\section{References}

[1] G.A. Rines, H.H. Zenzie, R.A. Schwarz, Y. Isyanova, P.F. Moulton, Nonlinear conversion of Ti:sapphire laser wavelengths, IEEE J. Sel. Top. Quant. Electron. 1 (1995) 50-57.

[2] S.G. Bartoshevich, I.V. Mikhnyuk, G.A. Skripko, I.G. Tarazevich, Efficient difference frequency oscillator based on a Ti:sapphire laser, IEEE J. Quant. Electron. 27 (1991) 2234-2237.

[3] H.H. Zenzie, P.E. Perkins, Temporal pulse synchronization for optical mixing, Tunable Solid State Laser Topical Meeting, North Falmouth, Cape Cod, MA, 1-3 May, 1989.

[4] M. Scheidt, K.-J. Boller, R. Wallenstein, Tunable noncritically phase-matched $\mathrm{cw}$ optical parametric oscillators of KTP, IEEE Laser and Electro-Optics Society Annual Meeting Conference Proceedings, vol. 2, 1994, pp. 334 335.

[5] H.H. Zenzie, P.F. Moulton, Tunable optical parametric oscillators pumped by Ti:sapphire laser, Opt. Lett. 19 (1994) 963-965.

[6] Ch. Grasser, Z. Marzenell, J. Dorring, R. Beugang, R. Wallenstein, in: S.A. Payne, C.R. Pollock (Eds.), OSA Trends in Optics and Photonics on Advanced Solid State Lasers, vol. 1, OSA, Washington, DC, 1996, pp. 158-163.

[7] J.Q. Yao, T.S. Fahlen, Calculations of optimum phase match parameters for the biaxial crystal $\mathrm{KTiOPO}_{4}$, J. Appl. Phys. 5 (1984) 65-68.

[8] J.Q. Yao, W.D. Sheng, W.Q. Shi, Accurate calculation of the optimum phase-matching parameters in three-wave interactions with biaxial nonlinear-optical crystals, J. Opt. Soc. Am. B 9 (1992) 891-902.

[9] A.V. Smith, SNLO Nonlinear Optics Code, Sandia National Laboratories.

[10] J.Q. Yao, Y. Zheng, Y. Tang, M.H. Dunn, W. Sibbett, The characteristics of sum-frequency-mixing by biaxial crystal $\mathrm{LiB}_{3} \mathrm{O}_{5}$ and $\mathrm{KiOPO}_{4}$, Conference on Laser and ElectroOptics/Pacific Rim Technical Digest, 1995, p. 111. 